\title{
Invariants of ideals generated by pfaffians
}

Emanuela De Negri. Università di Genova, Dipartimento di Matematica, Via Dodecaneso 35, IT-16146 Genova, Italia. email: denegri@dima.unige.it

Elisa Gorla. Universität Basel, Departement Mathematik, Rheinsprung 21, CH4051 Basel, Switzerland. email: elisa.gorla@unibas.ch

\begin{abstract}
Ideals generated by pfaffians are of interest in commutative algebra and algebraic geometry, as well as in combinatorics. In this article we compute multiplicity and Castelnuovo-Mumford regularity of pfaffian ideals of ladders. We give explicit formulas for some families of ideals, and indicate a procedure that allows to recursively compute the invariants of any pfaffian ideal of ladder. Our approach makes an essential use of liaison theory.
\end{abstract}

\section{Introduction}

Pfaffians are the natural analogue of minors when working with skew-symmetric matrices. Ideals generated by pfaffians are studied in the context of commutative algebra and algebraic geometry, as well as in combinatorics. Many are the reasons for such an interest, e.g., many ideals generated by pfaffians are Gorenstein (see, e.g., $[\mathrm{KL}]$ and $[\mathrm{D}]$ ). Conversely, due to a famous result ([BE]) of Buchsbaum and Eisenbud, any Gorenstein ideal of height 3 of a polynomial ring over a field is generated by the maximal pfaffians of a suitable skew-symmetric matrix of homogeneous forms. Ideals generated by pfaffians arise naturally in algebraic geometry as, e.g., ideals of pfaffians in a generic skew-symmetric matrix define Schubert cells in orthogonal Grassmannians. Moreover, some Grassmannians are defined by pfaffians, as well as some of their secant varieties.

In this article, we compute numerical invariants of pfaffian ideals of ladders. Pfaffian ideals of ladders are, informally speaking, ideals generated by pfaffians which only involve indeterminates in a ladder of a skew-symmetric matrix of indeterminates. The size of the pfaffians is allowed to vary in different regions of the ladder. This family was introduced by the authors in [DG, and contains the classically studied ideals of $2 t$-pfaffians of a matrix or of a ladder. It is a very large family, and a natural one to study from the point of view of liaison theory, since all the ideals in this family arise from ideals of $2 t$-pfaffians in a ladder while performing elementary G-biliaisons. In [DGo] we proved that these ideals are prime, normal and Cohen-Macaulay. The main result of the paper was a proof that any pfaffian ideal of ladder can be obtained from an ideal generated by indeterminates via a finite sequence of ascending G-biliaisons. In particular they are glicci, i.e., they belong to the G-liaison class of a complete intersection. The G-biliaison steps were described very explicitly. Therefore, as a biproduct, it is possible to recursively compute numerical invariants of pfaffian ideals of ladders such as the multiplicity, the Hilbert function, the $h$-vector, as well as a graded free resolution. In some cases it is also possible to compute the graded Betti numbers and in particular the Castelnuovo Mumford regularity. Although it is possible to perform these computations in any specific example, it is in general hard to produce explicit formulas. In this paper, we derive 
explicit formulas for some classes of pfaffian ideals of ladders.

The paper is organized as follows. In Section 1 we fix the notation and define the classes that we study. We also recall the main result of [DGo on which our approach is based. In Section 2 we give explicit or recursive formulas for the multiplicity of the ideals that we study. In Theorem 2.5 we give a simple numerical condition which forces the multiplicity of a pfaffian ideal of ladder to decompose as the product of the multiplicities of two pfaffian ideals relative to subladders. In Section 3 we compute Castelnuovo-Mumford regularities. In Section 4 we show how to use our approach to compute the graded Betti numbers of ideals of pfaffians of maximal size of a generic skew-symmetric matrix. We also give a simple proof that the $h$-vectors of these ideals are of decreasing type. The ideals generated by pfaffians of maximal size of a generic skew-symmetric matrix are Gorenstein ideals of height 3, so the results are well-known. However, we are able to give a very simple proof, which can be easily specialized to any Gorenstein ideal of height 3 .

Acknowledgements. The second author was supported by the Swiss National Science Foundation under grant no. 123393. Part of this work was done while the authors were attending the conference "PASI 2009 in Commutative Algebra and its Connections to Geometry, honoring Wolmer Vasconcelos", which took place in Olinda (Brazil) in August 2009. The authors wish to thank the organizers, the speakers and the participants to the conference for the stimulating working environment that they created.

\section{Some classes of pfaffian ladder ideals}

Let $X=\left(x_{i j}\right)$ be an $n \times n$ skew-symmetric matrix of indeterminates. In other words, the entries $x_{i j}$ with $i<j$ are indeterminates, $x_{i j}=-x_{j i}$ for $i>j$, and $x_{i i}=0$ for all $i=1, \ldots, n$. Let $R=K[X]=K\left[x_{i j} \mid 1 \leq i<j \leq n\right]$ be the polynomial ring associated to $X$.

Definition 1.1. A ladder $\mathcal{Y}$ of $X$ is a subset of the set $\left\{(i, j) \in \mathbb{N}^{2} \mid 1 \leq i, j \leq n\right\}$ with the following properties :

1. if $(i, j) \in \mathcal{Y}$ then $(j, i) \in \mathcal{Y}$,

2. if $i<h, j>k$ and $(i, j),(h, k)$ belong to $\mathcal{Y}$, then $(i, k),(i, h),(h, j),(j, k)$ belong to $\mathcal{Y}$.

We do not assume that a ladder $\mathcal{Y}$ is connected, nor that $X$ is the smallest skew-symmetric matrix having $\mathcal{Y}$ as ladder. We can assume without loss of generality that the ladder $\mathcal{Y}$ is symmetric.

It is easy to see that any ladder can be decomposed as a union of square subladders

$$
\mathcal{Y}=\mathcal{X}_{1} \cup \ldots \cup \mathcal{X}_{s}
$$

where

$$
\mathcal{X}_{k}=\left\{(i, j) \mid a_{k} \leq i, j \leq b_{k}\right\},
$$

for some integers $1 \leq a_{1} \leq \ldots \leq a_{s} \leq n$ and $1 \leq b_{1} \leq \ldots \leq b_{s} \leq n$ such that $a_{k}<b_{k}$ for all $k$. We say that $\mathcal{Y}$ is the ladder with upper corners $\left(a_{1}, b_{1}\right), \ldots,\left(a_{s}, b_{s}\right)$, and that $\mathcal{X}_{k}$ is the square subladder of $\mathcal{Y}$ with upper outside corner $\left(a_{k}, b_{k}\right)$. We allow two upper corners to have the same first or second coordinate, but we assume that no two upper corners coincide. Notice 
that with this convention a ladder does not have a unique decomposition of the form (1.1). In other words, a ladder does not correspond uniquely to a set of upper corners $\left(a_{1}, b_{1}\right), \ldots,\left(a_{s}, b_{s}\right)$. However, the upper corners determine the subladders $\mathcal{X}_{k}$, hence the ladder $\mathcal{Y}$ according to (1.1).

Let $t$ be a positive integer. A $2 t$-pfaffian is the pfaffian of a $2 t \times 2 t$ submatrix of $X$. Given a ladder $\mathcal{Y}$ we set $Y=\left\{x_{i j} \in X \mid(i, j) \in \mathcal{Y}, i<j\right\}$. We let $I_{2 t}(Y)$ denote the ideal generated by the set of the $2 t$-pfaffians of $X$ which involve only indeterminates of $Y$. In particular $I_{2 t}(X)$ is the ideal generated by the $2 t$-pfaffians of $X$. We regard all the ideals as ideals in $K[X]$.

Whenever we consider a ladder $\mathcal{Y}$, we assume that it comes with its set of upper corners and the corresponding decomposition as a union of square subladders as in 1.1).

The following family of ideals has been introduced and studied in DGo]:

Definition 1.2. Let $\mathcal{Y}=\mathcal{X}_{1} \cup \ldots \cup \mathcal{X}_{s}$ be a ladder as in Definition 1.1 Let $X_{k}=\left\{x_{i j} \mid(i, j) \in \mathcal{X}_{k}, i<j\right\}$ for $k=1, \ldots, s$. Fix a vector $\mathbf{t}=\left(t_{1}, \ldots, t_{s}\right)$, $\mathbf{t} \in\left\{1, \ldots,\left\lfloor\frac{n}{2}\right\rfloor\right\}^{s}$. The pfaffian ideal $I_{2 \mathbf{t}}(Y)$ is by definition the sum of pfaffian ideals $I_{2 t_{1}}\left(X_{1}\right)+\ldots+I_{2 t_{s}}\left(X_{s}\right) \subseteq K[X]$. We refer to these ideals as pfaffian ideals of ladders.

Remarks 1.3 (Remarks 1.5, DGo ). We can assume without loss of generality that

$$
2 t_{k} \leq b_{k}-a_{k}+1, \quad \text { for } 1 \leq k \leq s .
$$

Moreover, we can assume that

$$
a_{k}-a_{k-1}>t_{k-1}-t_{k} \text { and } \quad b_{k}-b_{k-1}>t_{k}-t_{k-1}
$$

for $2 \leq k \leq s$.

In DGo, pfaffian ideals of ladders are proved to be prime, normal, and Cohen-Macaulay. A formula for their height is given.

Notation 1.4. For a ladder $\mathcal{Y}$ with upper corners $\left(a_{1}, b_{1}\right), \ldots,\left(a_{s}, b_{s}\right)$ and $\mathbf{t}=$ $\left(t_{1}, \ldots, t_{s}\right)$, we denote by $\tilde{\mathcal{Y}}$ the ladder with upper corners $\left(a_{1}+t_{1}-1, b_{1}-t_{1}+\right.$ $1), \ldots,\left(a_{s}+t_{s}-1, b_{s}-t_{s}+1\right)$.

The ladder $\tilde{\mathcal{Y}}$ computes the height of the ideal $I_{2 \mathbf{t}}(Y)$ as follows:

Proposition 1.5 (Proposition 1.10, DGo ). Let $\mathcal{Y}$ be the ladder with upper corners $\left(a_{1}, b_{1}\right), \ldots,\left(a_{s}, b_{s}\right)$ and $\mathbf{t}=\left(t_{1}, \ldots, t_{s}\right)$. Let $\tilde{\mathcal{Y}}$ be as in Notation 1.4 . Then the height of $I_{2 \mathbf{t}}(Y)$ equals the cardinality of $\{(i, j) \in \tilde{\mathcal{Y}} \mid i<j\}$.

We now recall the definition of biliaison.

Definition 1.6. Let $I, I^{\prime}, J$ be homogeneous, saturated ideals in $K[X]$, with $\operatorname{ht}(I)=\operatorname{ht}\left(I^{\prime}\right)=\operatorname{ht}(J)+1$. Assume that $R / J$ is Cohen-Macaulay and generically Gorenstein, i.e., $(R / J)_{P}$ is Gorenstein for any minimal associated prime $P$ of $J$. We say that $I$ is obtained from $I^{\prime}$ by a G-biliaison of height $\ell$ on $J$ if $I / J$ and $I^{\prime} / J(\ell)$ represent the same element in the ideal class group of $K[X] / J$.

In other words, $I$ is obtained from $I^{\prime}$ by a G-biliaison of height $\ell$ on $J$ if there exist homogeneous polynomials $f, g \in R$ with $\operatorname{deg}(g)=\operatorname{deg}(f)+\ell$, such that $f I+J=g I^{\prime}+J$ as ideals of $R$.

The main result of DGo is that ladder pfaffian ideals belong to the Gbiliaison class of a complete intersection. In particular, they are glicci. We 
briefly recall the single G-biliaison step which is described in the proof of $\mathrm{DGO}$, Theorem 2.3]. With the notation of Definition [1.2, let $\mathcal{Y}^{\prime}$ be the subladder of $\mathcal{Y}$ with upper corners

$$
\left(a_{1}, b_{1}\right), \ldots,\left(a_{k-1}, b_{k-1}\right),\left(a_{k}+1, b_{k}-1\right),\left(a_{k+1}, b_{k+1}\right), \ldots,\left(a_{s}, b_{s}\right),
$$

and let $\mathbf{t}^{\prime}=\left(t_{1}, \ldots, t_{k-1}, t_{k}-1, t_{k+1}, \ldots, t_{s}\right)$. Let $\mathcal{Z}$ be the subladder of $\mathcal{Y}$ obtained by removing the entry $\left(a_{k}, b_{k}\right)$ and its symmetric. Equivalently, $\mathcal{Z}$ is the ladder with upper corners

$$
\left(a_{1}, b_{1}\right), \ldots,\left(a_{k-1}, b_{k-1}\right),\left(a_{k}, b_{k}-1\right),\left(a_{k}+1, b_{k}\right),\left(a_{k+1}, b_{k+1}\right), \ldots,\left(a_{s}, b_{s}\right) .
$$

Let $\mathbf{u}=\left(t_{1}, \ldots, t_{k-1}, t_{k}, t_{k}, t_{k+1}, \ldots, t_{s}\right)$. One has:

Theorem 1.7 (Theorem 2.3, DGo). Let $I=I_{2 \mathbf{t}}(Y), I^{\prime}=I_{2 \mathbf{t}^{\prime}}\left(Y^{\prime}\right)$ and $J=$ $I_{2 \mathbf{u}}(Z)$ be ideals of $K[X]$. Then $I$ is obtained from $I^{\prime}$ via an elementary $G$ biliaison of height 1 on $J$.

More precisely, with the above notation we have

$$
f I+J=g I^{\prime}+J
$$

where $f \in I^{\prime}$ is a $2\left(t_{k}-1\right)$-pfaffian, $g \in I$ is a $2 t_{k}$-pfaffian, and $f, g \notin J$.

When discussing biliaison, we will refer without distinction to the ideals and to the varieties associated to them.

In this paper we deal with special classes of pfaffian ideals of ladders, and we compute some of their numerical invariants using the biliaison step described in Theorem 1.7. The same technique gives a recursive procedure to determine such invariants for any pfaffian ideal of ladder. However, it is in general hard to deduce explicit formulas.

We now introduce the classes we are going to study. First we consider the ideal $L_{t}^{n}=I_{2 \mathbf{t}}(Y)$ where $\mathcal{Y}$ is the ladder with upper corners $(1, n-1)$ an $(2, n)$ and $\mathbf{t}=(t, t)$. Clearly $L_{t}^{n}$ is generated by the $2 t$-pfaffians of the ladder obtained from $X$ by deleting the entries $(1, n)$ and $(n, 1)$.

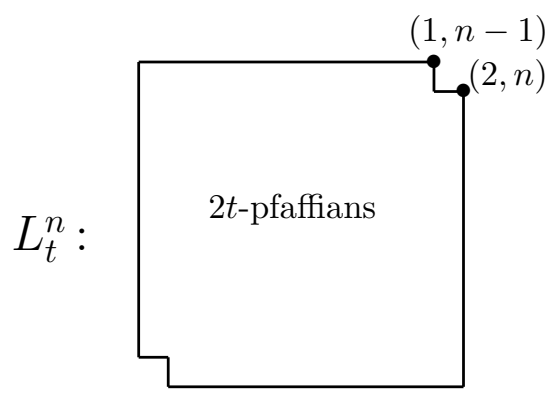

Then we restrict our attention to some ideals generated by pfaffians whose size is maximal or submaximal, in a sense that we are going to specify. In particular, we consider the ideals generated by maximal and by submaximal pfaffians of a skew-symmetric matrix of indeterminates. More precisely, we denote by $M_{t}$ the ideal generated by the $2 t$-Pfaffians of a $(2 t+1) \times(2 t+1)$ matrix and by $S M_{t}$ the ideal generated by the $2 t$-pfaffians of a $(2 t+2) \times(2 t+2)$ matrix.

Moreover we consider ideals generated by pfaffians of two different sizes in different regions of a matrix. Here we regard nested matrices as a ladder. In 
particular, we consider $N_{t}=I_{2 \mathbf{t}}(Y)$ where $\mathcal{Y}$ is the ladder with upper corners $(1,2 t-1)$ and $(1,2 t+1)$, and $\mathbf{t}=(t-1, t)$. So $N_{t}$ is the ideal generated by the $2 t$-pfaffians of a skew-symmetric matrix of size $2 t+1$ and the $(2 t-2)$-pfaffians of its first $2 t-1$ rows and columns. We denote by $S N_{t}$ the ideal $I_{2 \mathbf{t}}(Y)$ where $\mathcal{Y}$ is the ladder with upper corners $(1,2 t-1)$ and $(1,2 t+2)$, and $\mathbf{t}=(t-1, t)$. This is the ideal generated by the $2 t$-pfaffians of a skew-symmetric matrix of size $2 t+2$ and the $(2 t-2)$-pfaffians of its first $2 t-1$ rows and columns.

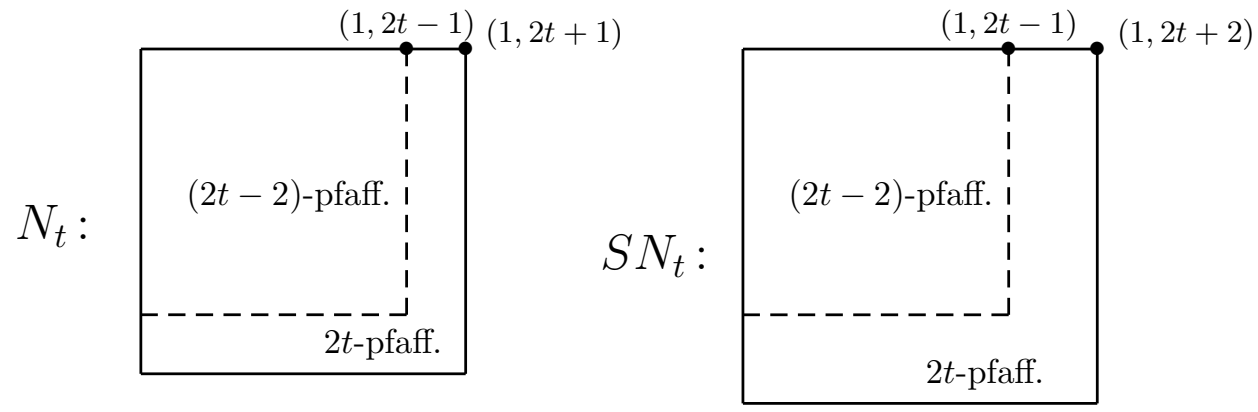

We let $L_{t}(k)=I_{2 \mathbf{t}}(Y)$, where $\mathcal{Y}$ is the ladder with upper corners $(1,2 t+$ $1),(2,2 t+2),(3,2 t+3), \ldots,(k, 2 t+k)$, and $\mathbf{t}=(t, \ldots, t)$. Notice that $L_{t}(1)=$ $M_{t}$, and $L_{t}(2)=L_{t}^{2 t+2}$.

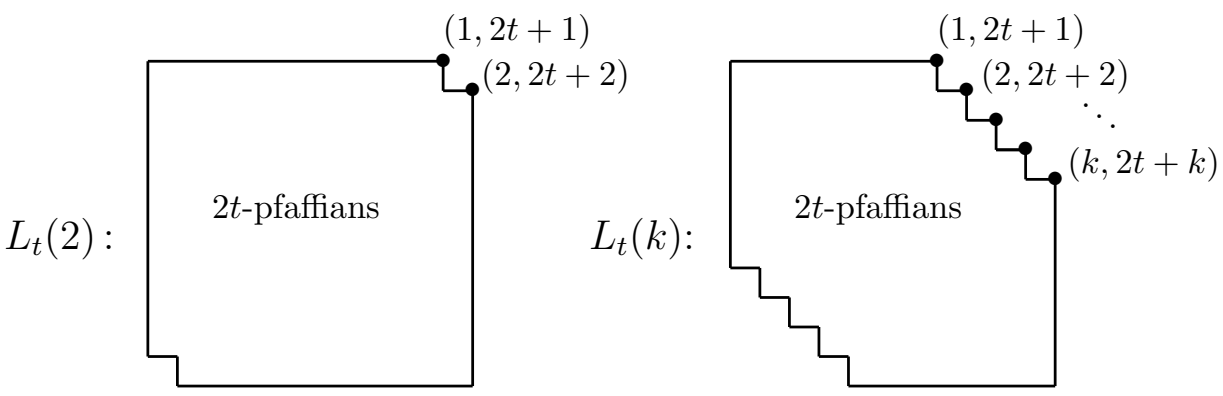

Moreover, given two integers $j$ and $k$ we let $\mathcal{Y}_{j k}$ be the ladder with the $j+k$ upper outside corners $(1,2 t-1),(2,2 t),(3,2 t+1), \ldots,(j, 2 t+j-2),(j, 2 t+$ $j),(j+1,2 t+j+1), \ldots,(j+k-1,2 t+j+k-1)$. We consider the ideal

$$
L_{t}(j, k):=I_{2 \mathbf{t}}\left(Y_{j k}\right), \text { where } \mathbf{t}=(\underbrace{t-1, \ldots, t-1}_{j}, \underbrace{t, \ldots, t}_{k}) .
$$

Notice that $L_{t}(0, k)=L_{t+1}(k, 0)$. Moreover, this class contains most of the classes that we have already introduced. More precisely: $L_{t}(k)=L_{t}(0, k)$, $M_{t}=L_{t}(0,1), S M_{t}=L_{t}(1,0)$, and $N_{t}=L_{t}(1,1)$.

Given two integers $j$ and $k$, we let $\mathcal{Z}_{j k}$ be the ladder with the $j+k$ upper outside corners $(1,2 t-1),(2,2 t),(3,2 t+1), \ldots,(j, 2 t+j-2),(j+1,2 t+j+$ $1), \ldots,(j+k, 2 t+j+k)$. We consider the ideal

$$
H_{t}(j, k):=I_{2 \mathbf{t}}\left(Z_{j k}\right), \text { where } \mathbf{t}=(\underbrace{t-1, \ldots, t-1}_{j}, \underbrace{t, \ldots, t}_{k}) .
$$

It is $L_{t}(k)=H_{t}(0, k)=H_{t+1}(k, 0)$. 

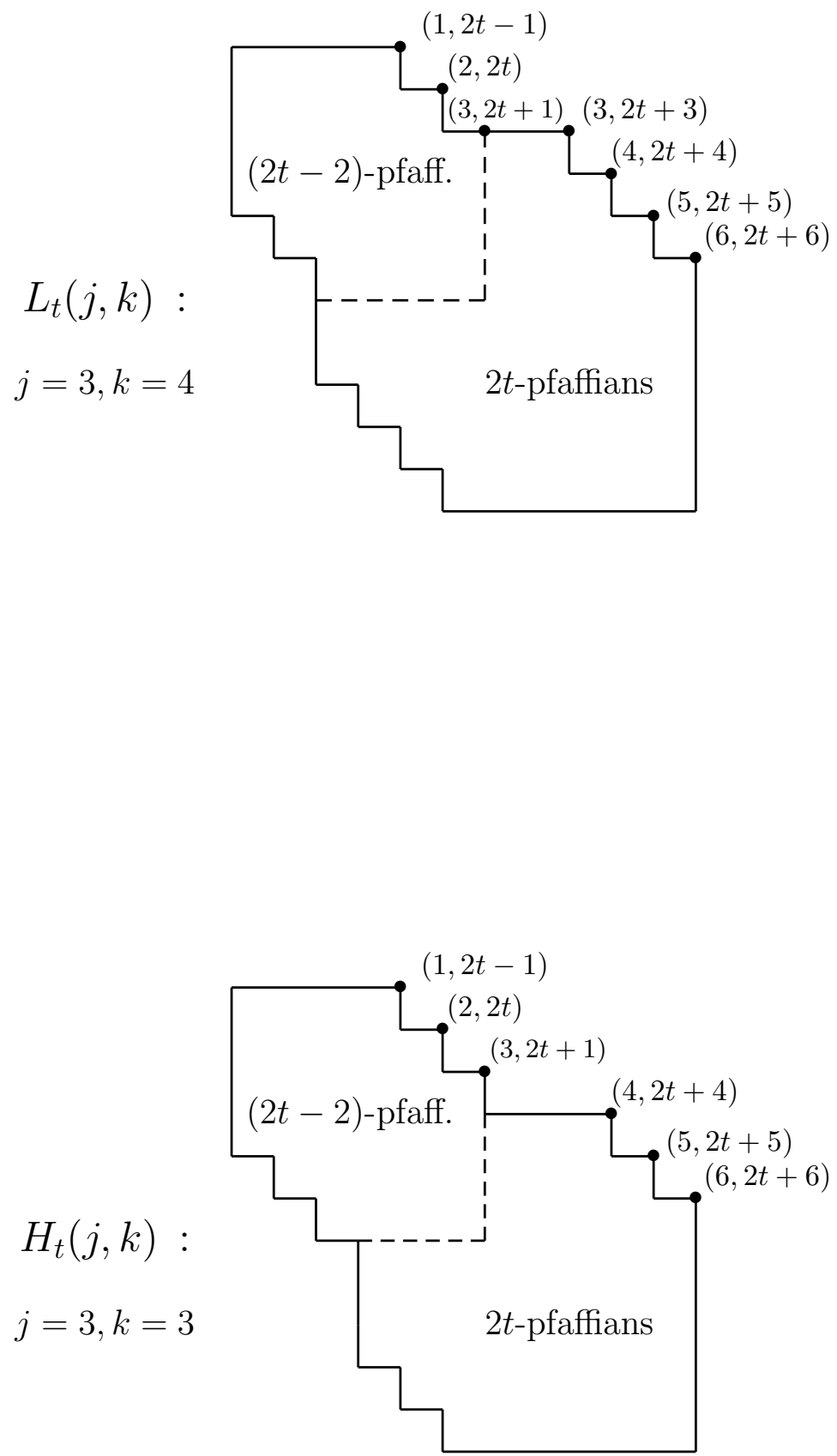


\section{Multiplicity of pfaffian ladder ideals}

In this section we give some formulas for the multiplicity of the ideals introduced in the previous section. Throughout the section, we denote by $e(I)$ the multiplicity of $R / I$ for any ideal $I \subset R=K[X]$. All the formulas that we produce are obtained as a finite sum of positive contributions. Therefore they are well suited to give lower bounds for the multiplicity. In the sequel we will need the following well know fact, which we prove for completeness.

Proposition 2.1. Let $H, I, J \subset K[X]$ be homogeneous, saturated, unmixed ideals. Assume that $H$ is Cohen-Macaulay and that $I$ is obtained from J via an elementary $G$-biliaison of height $\ell \in \mathbb{Z}$ on $H$. Then

$$
e(I)=e(J)+\ell e(H) .
$$

Proof. Let $U, S, T$ be the schemes associated to $H, I, J$, respectively. Under our assumptions, $U$ is arithmetically Cohen-Macaulay and $S, T$ are generalized divisors on $U$. Moreover, $S$ is linearly equivalent to $T+\ell h$ as generalized divisors on $U$, where $h$ denotes the hyperplane section class on $U$. In particular

$$
e(I)=\operatorname{deg}(S)=\operatorname{deg}(T)+\ell \operatorname{deg}(U)=e(J)+\ell e(H) .
$$

We denote by $I_{t}^{n}$ the ideal generated by the $2 t$-pfaffians of an $n \times n$ skewsymmetric matrix of indeterminates. In [K, Theorem 7] Krattenthaler proved that

$$
e\left(I_{t}^{n}\right)=\prod_{1 \leq i \leq j \leq n-2 t+1} \frac{2(t-1)+i+j}{i+j} .
$$

In particular for the ideals $M_{t}$ and $S M_{t}$ one has:

$$
e\left(M_{t}\right)=\prod_{1 \leq i \leq j \leq 2} \frac{2(t-1)+i+j}{i+j}, \quad e\left(S M_{t}\right)=\prod_{1 \leq i \leq j \leq 3} \frac{2(t-1)+i+j}{i+j} .
$$

From the results in DGo] one can easily deduce a formula for the multiplicity of the ideal $L_{t}^{n}$.

\section{Proposition 2.2.}

$e\left(L_{t}^{n}\right)=\frac{(n-2 t+2) !}{(2 n-4 t+4) !}\left[\frac{(2 n-2 t+2) !}{n !}-\frac{(n-1) !}{(2 t-3) !}\right] \prod_{1 \leq i \leq j \leq n-2 t+2} \frac{2(t-1)+i+j}{i+j}$

Proof. By Theorem 1.7 the ideal $I_{t}^{n+1}$ is obtained from $I_{t-1}^{n-1}$ via an elementary G-biliaison of height 1 on $L_{t}^{n}$. Hence by Proposition 2.1]

$$
e\left(L_{t}^{n}\right)=e\left(I_{t}^{n+1}\right)-e\left(I_{t-1}^{n-1}\right)
$$

Substituting (2.1) we obtain $e\left(L_{t}^{n}\right)=$

$$
\prod_{1 \leq i \leq j \leq n-2 t+2} \frac{1}{i+j}\left[\prod_{1 \leq i \leq j \leq n-2 t+2}(2 t-2+i+j)-\prod_{1 \leq i \leq j \leq n-2 t+2}(2 t-4+i+j)\right] .
$$

Since

$$
\prod_{1 \leq i \leq j \leq n-2 t+2}(2 t-4+i+j)=\prod_{0 \leq i \leq j \leq n-2 t+1}(2 t-2+i+j)
$$


by means of direct computation one gets

$$
\begin{aligned}
& \prod_{1 \leq i \leq j \leq n-2 t+2}(2 t-2+i+j)-\prod_{1 \leq i \leq j \leq n-2 t+1}(2(t-1)+i+j)\left[\prod_{1 \leq i \leq n-2 t+2}(2 t-4+i+j)=\right. \\
& \left.\prod_{1 \leq i \leq n-2 t+2}(n+i)-\prod_{0 \leq j \leq n-2 t+1}(2 t-2+j)\right]
\end{aligned}
$$

The result now follows from the equality

$$
\begin{gathered}
\prod_{1 \leq i \leq j \leq n-2 t+2} \frac{1}{i+j} \prod_{\substack{1 \leq i \leq j \leq n-2 t+1 \\
1 \leq i \leq j \leq n-2 t+1}}(2(t-1)+i+j)= \\
\prod_{i+j} \prod_{1 \leq i \leq n-2 t+2} \frac{1}{n-2 t+2+i} .
\end{gathered}
$$

The case of ideals generated by maximal pfaffians of a matrix has been extensively studied. In particular it is well known that

$$
e\left(L_{t}(1)\right)=e\left(M_{t}\right)=1+2^{2}+3^{2}+\cdots+t^{2}
$$

(see [HTV, Section 6], and [HT, Theorem 5.6 and the following example]).

We deduce the following formulas from Theorem 1.7.

\section{Proposition 2.3.}

$$
e\left(L_{t}(2)\right)=1+\sum_{s=2}^{t}\left[2 s\left(1+2^{2}+\cdots+s^{2}\right)-s^{3}\right]
$$

and

$$
e\left(N_{t}\right)=1+\sum_{s=2}^{t-1}\left[2 s\left(1+2^{2}+\cdots+s^{2}\right)-s^{3}\right]+t\left(1+2^{2}+\cdots+(t-1)^{2}\right) .
$$

Proof. By Theorem 1.7 the ideal $L_{t}(2)$ is obtained from $N_{t}$ via an elementary G-biliaison of height 1 on $M_{t}+(f)$, where $f$ is a $2 t$-pfaffian which is regular modulo $M_{t}$. Thus by Proposition 2.1 one has

$$
e\left(L_{t}(2)\right)=e\left(N_{t}\right)+e\left(M_{t}+(f)\right)=e\left(N_{t}\right)+t e\left(M_{t}\right) .
$$

Moreover the ideal $N_{t}$ is obtained from $L_{t-1}(2)$ via an elementary G-biliaison of height 1 on $M_{t-1}+(g)$, where $g$ is a $2 t$-pfaffian which is regular modulo $M_{t-1}$. Therefore

$$
e\left(N_{t}\right)=e\left(L_{t-1}(2)\right)+t e\left(M_{t-1}\right)
$$

and combining (2.3) and (2.4) one gets

$$
e\left(L_{t}(2)\right)=e\left(L_{t-1}(2)\right)+t e\left(M_{t-1}\right)+t e\left(M_{t}\right) .
$$

Finally by (2.5) and (2.2), after solving the recursion one obtains

$$
e\left(L_{t}(2)\right)=1+\sum_{s=2}^{t}\left[s\left(e\left(M_{s-1}\right)+e\left(M_{s}\right)\right)\right]=1+\sum_{s=2}^{t}\left[2 s\left(1+2^{2}+\cdots+s^{2}\right)-s^{3}\right] .
$$

The formula for $e\left(N_{t}\right)$ follows from substituting the formula for $e\left(L_{t}(2)\right)$ and (2.2) in (2.4). 
We now deduce a formula for the multiplicity of ideals generated by submaximal pfaffians.

Corollary 2.4.

$$
e\left(S M_{t}\right)=t+\sum_{r=2}^{t} \sum_{s=2}^{r}\left[2 s\left(1+2^{2}+\cdots+s^{2}\right)-s^{3}\right] .
$$

Proof. Since $S M_{t}$ is obtained from $S M_{t-1}$ via an elementary G-biliaison of height 1 on $L_{t}(2)$, one has $e\left(S M_{t}\right)=e\left(S M_{t-1}\right)+e\left(L_{t}(2)\right)$. By solving the recursion and using Proposition 2.3. one obtains the result.

Let $\mathcal{Y}=\mathcal{Y}_{1} \cup \mathcal{Y}_{2}$ be a ladder which is union of two smaller ladders. Let $I_{1}=I_{2 \mathbf{t}_{1}}\left(Y_{1}\right)$ and $I_{2}=I_{2 \mathbf{t}_{\mathbf{2}}}\left(Y_{2}\right)$ be pfaffian ideals associated to the ladders $\mathcal{Y}_{1}$ and $\mathcal{Y}_{2}$, and let the upper corners of $\mathcal{Y}$ be the union of the upper corners of $\mathcal{Y}_{1}$ and $\mathcal{Y}_{2}$. Let $\mathbf{t}=\mathbf{t}_{\mathbf{1}} \oplus \mathbf{t}_{\mathbf{2}}$ be the vector obtained by appending the vector $\mathbf{t}_{\mathbf{2}}$ to the vector $\mathbf{t}_{\mathbf{1}}$ and let $I=I_{2 t}(Y)=I_{1}+I_{2}$ be the pfaffian ideal associated to the ladder $\mathcal{Y}$. If $\mathcal{Y}_{1} \cap \mathcal{Y}_{2}=\emptyset$, one can easily show that

$$
e(I)=e\left(I_{1}\right) e\left(I_{2}\right) .
$$

The following theorem gives a sufficient condition on the ladder so that (2.6) holds.

Theorem 2.5. Let $\mathcal{Y}, \mathcal{Y}_{1}, \mathcal{Y}_{2}$ be ladders, $\mathcal{Y}=\mathcal{Y}_{1} \cup \mathcal{Y}_{2}$. Let $I_{1}=I_{2 \mathbf{t}_{1}}\left(Y_{1}\right)$ and $I_{2}=I_{2 \mathbf{t}_{2}}\left(Y_{2}\right)$ be pfaffian ideals of ladders associated to $\mathcal{Y}_{1}$ and $\mathcal{Y}_{2}$. Let $\mathbf{t}=\mathbf{t}_{\mathbf{1}} \oplus \mathbf{t}_{\mathbf{2}}$ and let $I=I_{2 \mathbf{t}}(Y)=I_{1}+I_{2}$ be the corresponding pfaffian ideal of ladder. Let $\tilde{\mathcal{Y}}, \tilde{\mathcal{Y}}_{1}, \tilde{\mathcal{Y}}_{2}$ be defined as in Notation 1.4 and let $\tilde{Y}, \tilde{Y}_{1}, \tilde{Y}_{2}$ be the corresponding sets of indeterminates. If $\tilde{Y}_{1} \cap \tilde{Y}_{2}=\emptyset$, then

$$
e(I)=e\left(I_{1}\right) e\left(I_{2}\right) .
$$

Proof. Let $\mathcal{Z}=\mathcal{Y}_{1} \cap \mathcal{Y}_{2}, R_{1}=K\left[Y_{1}\right] / I_{1}$, and $R_{2}=K\left[Y_{2}\right] / I_{2}$. We have

$$
K[Y] / I \cong R_{1} \otimes_{K} R_{2} / J
$$

where $J$ is generated by $|Z|$ linear forms (which identify the corresponding indeterminates in $Y_{1}$ and $Y_{2}$ ). If $\tilde{Y}_{1} \cap \tilde{Y}_{2}=\emptyset$, then

$$
\text { ht } I=\text { ht } I_{1}+\text { ht } I_{2}
$$

hence

ht $J=\operatorname{dim} R_{1} \otimes R_{2}-\operatorname{dim} K[Y] / I=\left|Y_{1}\right|-$ ht $I_{1}+\left|Y_{2}\right|-$ ht $I_{2}-|Y|+$ ht $I=|Z|$.

Since $R_{1} \otimes R_{2}$ is a Cohen-Macaulay ring, $J$ is generated by a regular sequence and

$$
e(I)=e\left(R_{1} \otimes_{K} R_{2} / J\right)=e\left(I_{1}\right) e\left(I_{2}\right) .
$$

We now give an example of a family of pfaffian ideals of ladders whose multiplicity can be computed directly from Theorem 2.5

Proposition 2.6.

$$
e\left(H_{t}(j, k)\right)=e\left(L_{t-1}(j)\right) e\left(L_{t}(k)\right) .
$$


Proof. Let $\mathcal{Y}=\mathcal{Z}_{j k}$ be the ladder with the $j+k$ upper corners $(1,2 t-1),(2,2 t)$, $\ldots,(j, 2 t+j-2),(j+1,2 t+j+1), \ldots,(j+k, 2 t+j+k)$. Let $\mathcal{Y}_{1}$ be the ladder with the $j$ upper corners $(1,2 t-1), \ldots,(j, 2 t+j-2)$ and let $\mathcal{Y}_{2}$ be the ladder with the $k$ upper corners $(j+1,2 t+j+1), \ldots,(j+k, 2 t+j+k)$. Clearly $\mathcal{Y}=\mathcal{Y}_{1} \cup \mathcal{Y}_{2}$. Let

$$
\mathbf{t}_{\mathbf{1}}=(\underbrace{t-1, \ldots, t-1}_{j}), \mathbf{t}_{\mathbf{2}}=(\underbrace{t, \ldots, t}_{k}), \mathbf{t}=\mathbf{t}_{\mathbf{1}} \oplus \mathbf{t}_{\mathbf{2}}=(\underbrace{t-1, \ldots, t-1}_{j}, \underbrace{t, \ldots, t}_{k}) .
$$

Then $\tilde{\mathcal{Y}}_{1}$ is the ladder with upper outside corners $(t-1, t+1),(t, t+2), \ldots,(t+j-$ $2, t+j)$ and $\tilde{\mathcal{Y}}_{2}$ is the ladder with upper outside corners $(t+j, t+j+2), \ldots,(t+$ $j+k-1, t+j+k+1)$. Hence $\tilde{\mathcal{Y}}_{1} \cap \tilde{\mathcal{Y}}_{2}=\{(t+j, t+j)\}$ and $Y_{1} \cap Y_{2}=\emptyset$. By Theorem 2.5 it follows that

$$
e\left(H_{t}(j, k)\right)=e\left(I_{1}\right) e\left(I_{2}\right)
$$

where $I_{1}=I_{2 \mathbf{t}_{1}}\left(Y_{1}\right)$ and $I_{2}=I_{2 \mathbf{t}_{2}}\left(Y_{2}\right)$. The thesis follows from the observation that $I_{1}=L_{t-1}(j)$ and $I_{2}=L_{t}(k)$.

Combining Proposition 2.6 and Proposition 2.8, we obtain a formula for the multiplicity of the ideals $L_{t}(j, k)$.

Proposition 2.7. For $j, k \geq 1$ we have

$e\left(L_{t}(j, k)\right)=e\left(L_{t-1}(j+k)\right)+t e\left(L_{t-1}(j+k-1)\right)+\sum_{l=1}^{k-1} e\left(L_{t-1}(j+k-1-l)\right) e\left(L_{t}(l)\right)$.

Proof. We proceed by induction on $k \geq 1$. By Theorem 1.7 $L_{t}(j, 1)$ is obtained from $L_{t-1}(j+1)$ via an elementary G-biliaison on $L_{t-1}(j)+(f)$, where $f$ is a $2 t$-pfaffian which does not belong to $L_{t-1}(j)$. Hence by Proposition 2.1

$$
e\left(L_{t}(j, 1)\right)=e\left(L_{t-1}(j+1)\right)+t e\left(L_{t-1}(j)\right) .
$$

This proves the thesis for $k=1$. To establish the formula for $k \geq 2$, observe that $L_{t}(j, k)$ is obtained from $L_{t}(j+1, k-1)$ via an elementary G-biliaison of height 1 on $H_{t}(j, k-1)$. Hence by Proposition 2.1 and Proposition 2.6

$$
e\left(L_{t}(j, k)\right)=e\left(L_{t}(j+1, k-1)\right)+e\left(L_{t-1}(j)\right) e\left(L_{t}(k-1)\right) .
$$

By induction hypothesis $e\left(L_{t}(j+1, k-1)\right)=$

$$
e\left(L_{t-1}(j+k)\right)+t e\left(L_{t-1}(j+k-1)\right)+\sum_{l=1}^{k-2} e\left(L_{t-1}(j+k-1-l)\right) e\left(L_{t}(l)\right)
$$

and the thesis follows.

Explicit formulas for $e\left(L_{t}(1)\right)$ and $e\left(L_{t}(2)\right)$ were given in (2.2) and in Proposition 2.3. Since $L_{1}(k)$ is generated by indeterminates, $e\left(L_{1}(k)\right)=1$ for any $k$. The following formula allows us to calculate $e\left(L_{t}(k)\right)$ recursively, for $t \geq 2$ and $k \geq 3$.

Proposition 2.8. For $t, k \geq 2$ we have $e\left(L_{t}(k)\right)=$

$$
e\left(L_{t-1}(k)\right)+t\left[e\left(L_{t}(k-1)\right)+e\left(L_{t-1}(k-1)\right)\right]+\sum_{l=1}^{k-2} e\left(L_{t-1}(k-1-l)\right) e\left(L_{t}(l)\right) .
$$


Proof. By Theorem 1.7 $L_{t}(k)$ is obtained from $L_{t}(1, k-1)$ via an elementary G-biliaison of height 1 on $L_{t}(k-1)+(f)$, where $f$ is a $2 t$-pfaffian which does not belong to $L_{t}(k-1)$. Hence by Proposition 2.1 and Proposition 2.7

$$
\begin{gathered}
e\left(L_{t}(k)\right)=L_{t}(1, k-1)+t e\left(L_{t}(k-1)\right)= \\
e\left(L_{t-1}(k)\right)+t\left[e\left(L_{t}(k-1)\right)+e\left(L_{t-1}(k-1)\right)\right]+\sum_{l=1}^{k-2} e\left(L_{t-1}(k-1-l)\right) e\left(L_{t}(l)\right) .
\end{gathered}
$$

Remarks 2.9. 1. Proposition 2.8 allows us to compute the multiplicity of the ideals $L_{t}(k)$ for any values of $t$ and $k$. This can in fact be done recursively, using as a starting point that $e\left(L_{1}(k)\right)=1$ for any $k$, and the explicit formulas for the multiplicities of $L_{t}(1)=M_{t}$ and $L_{t}(2)$ which appear in (2.2) and in Proposition 2.3, respectively.

2. Proposition 2.7 allows us to compute the multiplicity of the ideals $L_{t}(j, k)$ for any values of $t, j, k$. One can in fact use Proposition 2.8 to compute the multiplicities of $L_{t}(1), \ldots, L_{t}(k-1)$ and $L_{t-1}(j), \ldots, L_{t-1}(j+k)$.

3. Since $L_{t}(k)=L_{t}(0, k)$, the multiplicity of $L_{t}(j, k)$ for $j=0$ is computed in Proposition 2.8. In fact, the formula obtained in Proposition 2.8 corresponds to the formula computed in Proposition 2.7 for $j=0$, taken "cum grano salis".

4. The formula given in Proposition 2.7 is false for $k=0$.

Finally, we express the multiplicity of $S N_{t}$ in terms of the multiplicities of $S M_{t}$ and $L_{t}(1,2)$. The latter two can be computed by Proposition 2.4 and Proposition 2.7.

Proposition 2.10. For $t \geq 1$ we have

$$
e\left(S N_{t}\right)=\sum_{s=2}^{t} e\left(L_{s}(1,2)\right)+\sum_{s=2}^{t-1} s e\left(S M_{s}\right)+1 .
$$

Proof. We proceed by induction on $t$. If $t=1$, then $S N_{1}$ is generated by indeterminates and $e\left(S N_{1}\right)=1$.

Let $\mathcal{Y}$ denote the ladder with upper corners $(1,2 t-1)$ and $(2,2 t+1)$. Then $I_{2(t-1)}(Y)$ is the ideal generated by the $2(t-1)$-pfaffians of $\mathcal{Y}$. By Theorem 1.7 $S N_{t}$ is obtained from $I_{2(t-1)}(Y)$ via an elementary G-biliaison of height 1 on $L_{t}(1,2)$. In turn, $I_{2(t-1)}(Y)$ is obtained from $S N_{t-1}$ via an elementary Gbiliaison of height 1 on $S M_{t-1}+(f)$, where $f$ is a $2(t-1)$-pfaffian which does not belong to $S M_{t-1}$. Therefore, by Proposition 2.1

$$
e\left(S N_{t}\right)=e\left(L_{t}(1,2)\right)+(t-1) e\left(S M_{t-1}\right)+e\left(S N_{t-1}\right)
$$

and the thesis follows by induction hypothesis.

Remark 2.11. From the proof of Proposition 2.10 it also follows that

$$
e\left(I_{2(t-1)}(Y)\right)=e\left(S N_{t}\right)-e\left(L_{t}(1,2)\right)=\sum_{s=2}^{t-1}\left[e\left(L_{s}(1,2)+s e\left(M_{s}\right)\right]+1 .\right.
$$




\section{Castelnuovo-Mumford regularity}

In this section we use biliaison to compute the Castelnuovo-Mumford regularity of some of the ideals considered in the previous section. For an ideal $I$ of $R=K[X]$, we denote by $\beta_{i, j}(I)$ the $(i, j)$ - th graded Betti number of $I$, regarded as an $R$-module. The Castelnuovo-Mumford regularity of a Cohen-Macaulay ideal $I$ of height $h=\operatorname{ht}(I)$ is

$$
\operatorname{reg}(I)=\max \left\{j \mid \beta_{h-1, j}(I) \neq 0\right\}-h+1 .
$$

It is well known that $\operatorname{reg}\left(M_{t}\right)=2 t-1$.

The following result allows us to recursively compute the Castelnuovo Mumford regularities of ideals obtained one from the other by biliaison.

Theorem 3.1. Let $H, I, J \subset R$ be homogeneous, Cohen-Macaulay ideals. Assume that $I$ is obtained from $J$ via an elementary G-biliaison of height $\ell \in \mathbb{Z}$ on $H$. If $\operatorname{reg}(J)<\operatorname{reg}(H)$, then

$$
\operatorname{reg}(I)=\operatorname{reg}(H)+\ell-1
$$

Proof. Since $I$ is obtained from $J$ via an elementary G-biliaison of height $\ell \in \mathbb{Z}$ on $H$, there are homogeneous polynomials $f, g$ with $\operatorname{deg}(f)+\ell=\operatorname{deg}(g)=: t$ such that

$$
f I+H=g J+H \subset R .
$$

Let $h=$ ht $I=$ ht $J=$ ht $H+1$. Applying the Mapping Cone construction to the short exact sequence

$$
0 \longrightarrow H[-t] \longrightarrow H \oplus J[-t] \longrightarrow g J+H \longrightarrow 0
$$

we have that

$$
\begin{gathered}
\operatorname{reg}(g J+H)=\max \left\{j \mid \beta_{h-1, j}(g J+H) \neq 0\right\}-h+1= \\
\max \{\operatorname{reg}(H)+h-2, \operatorname{reg}(J)+h-1\}+t-h+1=\operatorname{reg}(H)+t-1 .
\end{gathered}
$$

The last equality follows from the assumption that $\operatorname{reg}(J)<\operatorname{reg}(H)$. The previous equality follows from the observation that, since $J$ and $H$ are CohenMacaulay ideals,

$$
\begin{gathered}
\max \left\{j \mid \beta_{h-2, j}(H) \neq 0\right\}=\operatorname{reg}(H)+h-2 \geq \\
\operatorname{reg}(J)+h-1>\max \left\{j \mid \beta_{h-2, j}(J) \neq 0\right\}
\end{gathered}
$$

therefore no cancellation involving a direct summand $R[-r e g(H)+h-2]$ can take place in the free resolution of $g J+H$.

In an analogous fashion, we can produce a free resolution for $g J+H=f I+H$ by applying the Mapping Cone construction to the short exact sequence

$$
0 \longrightarrow H[-t+\ell] \longrightarrow H \oplus I[-t+\ell] \longrightarrow f I+H \longrightarrow 0 .
$$

Since

$$
\begin{gathered}
\max \left\{j \mid \beta_{h-1, j}(f I+H) \neq 0\right\}=\operatorname{reg}(H)+t+h-2> \\
\operatorname{reg}(H)+h-2+t-\ell=\max \left\{j \mid \beta_{h-2, j}(H[-t+\ell]) \neq 0\right\},
\end{gathered}
$$

it must be

$$
\operatorname{reg}(H)+t+h-2=\max \left\{j \mid \beta_{h-1, j}(I[-t+\ell]) \neq 0\right\}=\operatorname{reg}(I)+h-1+t-\ell,
$$

hence

$$
\operatorname{reg}(I)=\operatorname{reg}(H)+\ell-1
$$


We now derive formulas for the Castelnuovo-Mumford regularity of some pfaffian ideals of ladders. They are all easy consequences of Theorem 3.1 .

Proposition 3.2. For $t \geq 1$ we have

$$
\operatorname{reg}\left(L_{t}(2)\right)=3 t-2
$$

and for $t \geq 2$

$$
\operatorname{reg}\left(N_{t}\right)=3 t-4 \text {. }
$$

Proof. We compute the regularity of $L_{t-1}(2)$ and $N_{t}$ for $t \geq 2$. We proceed by induction on $t \geq 2$. If $t=2, L_{1}(2)$ is generated by indeterminates, hence $\operatorname{reg}\left(L_{1}(2)\right)=1$. By Theorem 1.7, $N_{2}$ is obtained from $L_{1}(2)$ via an ascending G-biliaison of height 1 on $M_{1}+(p)$, where $p$ is a 4 -pfaffian which is regular modulo $M_{1}$. Since $\operatorname{reg}\left(L_{1}(2)\right)=1<2=\operatorname{reg}(H)$, by Theorem 3.1 we have

$$
\operatorname{reg}\left(N_{2}\right)=2 \text {. }
$$

We now assume by induction hypothesis that $\operatorname{reg}\left(L_{t-2}(2)\right)=3 t-8$ and $\operatorname{reg}\left(N_{t-1}\right)=3 t-7$, and compute the regularity of $L_{t-1}(2)$ and $N_{t}$. By Theorem 1.7. the ideal $L_{t-1}(2)$ is obtained from $N_{t-1}$ via an elementary G-biliaison of height 1 on $M_{t-1}+(f)$, where $f$ is a $2(t-1)$-pfaffian which is regular modulo $M_{t-1}$. Since $\operatorname{reg}\left(N_{t-1}\right)=3 t-7<3 t-5=\operatorname{reg}\left(M_{t-1}+(f)\right)$, by Theorem 3.1

$$
\operatorname{reg}\left(L_{t-1}(2)\right)=3 t-5 .
$$

By Theorem 1.7 the ideal $N_{t}$ is obtained from $L_{t-1}(2)$ via an elementary Gbiliaison of height 1 on $M_{t-1}+(g)$, where $g$ is a $2 t$-pfaffian which is regular modulo $M_{t-1}$. Since $\operatorname{reg}\left(L_{t-1}(2)\right)=3 t-5<3 t-4=\operatorname{reg}\left(M_{t-1}+g\right)$, by Theorem 3.1 we have

$$
\operatorname{reg}\left(N_{t}\right)=3 t-4
$$

Proposition 3.3. For $t \geq 1$ we have

$$
\operatorname{reg}\left(S M_{t}\right)=3 t-2
$$

Proof. We proceed by induction on $t \geq 1$. If $t=1, S M_{1}$ is generated by indeterminates, hence $\operatorname{reg}\left(S M_{1}\right)=1$. By Theorem 1.7 the ideal $S M_{t}$ is obtained from $S M_{t-1}$ via an elementary G-biliaison of height 1 on $L_{t}(2)$. By induction hypothesis and Proposition 3.2

$$
\operatorname{reg}\left(S M_{t-1}\right)=3 t-5<3 t-2=\operatorname{reg} L_{t}(2) .
$$

Therefore, by Theorem 3.1

$$
\operatorname{reg}\left(S M_{t}\right)=\operatorname{reg}\left(L_{t}(2)\right)=3 t-2 .
$$




\section{The Gorenstein height 3 case}

The ideal $M_{t}$ generated by the $2 t$-pfaffians of a generic skew-symmetric matrix of size $2 t+1$ is a Gorenstein ideal of height 3. A classical result due to Buchsbaum and Eisenbud $\mathrm{BE}$ states that any Gorenstein ideal of height 3 is obtained by specialization from $M_{t}$, for some $t$. An alternative proof for many classically known results on Gorenstein ideals of height 3 can therefore be given by combining specialization with a liaison approach analogous to what we have done in the previous sections.

In this section we wish to give a taste of what can be obtained following such an approach. In particular, we use G-biliaison to compute the graded Betti numbers of the ideal $M_{t}$ and to prove that its $h$-vector is of decreasing type. We start by recalling some definitions and fixing the notation.

Let $I$ be a homogeneous ideal of $R=K[X]$. The Hilbert function of $R / I$ is defined as

$$
\mathrm{H}_{I}(m)=\operatorname{dim}_{K}(R / I)_{m}
$$

for every integer $m$. Clearly $\mathrm{H}_{I}(m)=0$ for $m<0$. The formal power series

$$
\operatorname{HS}_{I}(z)=\sum_{m \in \mathbb{Z}} \mathrm{H}_{I}(m) z^{m}
$$

is called the Hilbert series of $R / I$. It is well known that the Hilbert series of $R / I$ is of the form

$$
\operatorname{HS}_{I}(z)=\frac{h_{I}(0)+h_{I}(1) z+\ldots+h_{I}(s) z^{s}}{(1-z)^{d}},
$$

where $d=\operatorname{dim}(R / I)$ and $h_{i} \in \mathbb{Z}$ for every $i$. The vector

$$
h_{I}=\left(h_{I}(0), \ldots, h_{I}(s)\right) \in \mathbb{Z}^{s}
$$

is called $h$-vector of $I$. Moreover we denote by $\Delta H_{I}$ the first difference of $H_{I}$, that is

$$
\Delta H_{I}(m)=H_{I}(m)-H_{I}(m-1) .
$$

Definition 4.1. Let $h=\left(h_{0}, h_{1}, \ldots, h_{s}\right) \in \mathbf{Z}^{s}$

a) $h$ is unimodal if there exists $t \in\{1, \ldots s\}$ such that $h_{1} \leq h_{2} \leq \cdots \leq h_{t} \geq$ $h_{t+1} \geq \cdots \geq h_{s}$.

b) $h$ is of decreasing type if whenever $h_{t}>h_{t+1}$, then $h_{j}>h_{j+1}$ for every $j>t$.

Notice that every $h$-vector of decreasing type is unimodal.

Proposition 4.2. The h-vector of $M_{t}$ is of decreasing type.

Proof. Let $X$ be a $(2 t+1) \times(2 t+1)$ skew-symmetric matrix of indeterminates and let $R=K[X]$ be the corresponding polynomial ring. Denote by $h_{(t, t)}(m)$ the $m$-th entry of the $h$-vector of a complete intersection generated by two forms of degree $t$.

We follow the notation of Section 1 and consider the ideals $M_{t}, M_{t-1}$ and $L_{t}^{2 t+1}=: I$. It is clear that $I$ is generated by two $2 t$-pfaffians which form a complete intersection. By Theorem 1.7 $M_{t-1}$ is obtained from $M_{t}$ via an elementary G-biliaison of height 1 on $I$. In other words, there are homogeneous polynomials $f, g$ of degree $t-1$ and $t$ respectively, such that

$$
f M_{t}+I=g M_{t-1}+I \subset R .
$$


By the additivity of the Hilbert function on the two short exact sequences

$$
\begin{gathered}
0 \longrightarrow I[-t+1] \longrightarrow I \oplus M_{t}[-t+1] \longrightarrow f M_{t}+I \longrightarrow 0 \\
0 \longrightarrow I[-t] \longrightarrow I \oplus M_{t-1}[-t] \longrightarrow g M_{t-1}+I \longrightarrow 0
\end{gathered}
$$

one obtains that $H_{M_{t}}(d-t+1)-H_{I}(d-t+1)=H_{M_{t-1}}(d-t)-H_{I}(d-t)$ for any $d \in \mathbb{Z}$. By setting $m=d-t+1$, we get

$$
H_{M_{t}}(m)=H_{M_{t-1}}(m-1)+\Delta H_{I}(m) .
$$

Since $\operatorname{dim} R / I-1=\operatorname{dim} R / M_{t-1}=\operatorname{dim} R / M_{t}$, one has

$$
h_{M_{t}}(m)=h_{M_{t-1}}(m-1)+h_{(t, t)}(m) .
$$

Solving the recursion one obtains

$$
h_{M_{t}}(m)=h_{M_{1}}(m-t+1)+\sum_{j=2}^{t} h_{(j, j)}(m-t+j) .
$$

This proves that the $h$-vector of $M_{t}$ is obtained by summing the $h$-vectors of suitable complete intersections. Notice that the $h$-vectors involved in the summation are shifted in such a way, that the maximum is always attained at the same point. Therefore, their sum $h_{M_{t}}$ is of decreasing type.

We can easily compute the graded Betti numbers of $M_{t}$ as follows.

Proposition 4.3. A minimal free resolution of $M_{t}$ has the form

$$
0 \longrightarrow R[-2 t-1] \longrightarrow R[-t-1]^{2 t+1} \longrightarrow R[-t]^{2 t+1} \longrightarrow M_{t} \longrightarrow 0 .
$$

Proof. We prove the statement by induction on $t \geq 1$. If $t=1$, the ideal $M_{1}$ is generated by three distinct indeterminates, hence a minimal free resolution has the form

$$
0 \longrightarrow R[-3] \longrightarrow R[-2]^{3} \longrightarrow R[-1]^{3} \longrightarrow M_{1} \longrightarrow 0 .
$$

Assume now that $t \geq 2$ and consider the ideals $M_{t}, M_{t-1}$ and $L_{t}^{2 t+1}$. We denote $L_{t}^{2 t+1}$ by $I$ for brevity. It is clear that $I$ is generated by two $2 t$-pfaffians which form a complete intersection. By Theorem [1.7, $M_{t-1}$ is obtained from $M_{t}$ via an elementary G-biliaison of height 1 on $I$. Moreover, there are homogeneous polynomials $f, g$ of degree $t-1$ and $t$ respectively, such that

$$
f M_{t}+I=g M_{t-1}+I \subset R .
$$

By induction hypothesis $M_{t-1}$ has a minimal free resolution of the form

$$
0 \longrightarrow R[-2 t+1] \longrightarrow R[-t]^{2 t-1} \longrightarrow R[-t+1]^{2 t-1} \longrightarrow M_{t-1} \longrightarrow 0 .
$$

Let

$$
0 \longrightarrow \mathbb{F}_{3} \longrightarrow \mathbb{F}_{2} \longrightarrow \mathbb{F}_{1} \longrightarrow M_{t} \longrightarrow 0
$$

be a minimal free resolution of $M_{t}$. Applying the Mapping Cone to the two short exact sequences

$$
\begin{gathered}
0 \longrightarrow I[-t+1] \longrightarrow I \oplus M_{t}[-t+1] \longrightarrow f M_{t}+I \longrightarrow 0 \\
0 \longrightarrow I[-t] \longrightarrow I \oplus M_{t-1}[-t] \longrightarrow g M_{t-1}+I \longrightarrow 0
\end{gathered}
$$


one obtains free resolutions for the ideal $J=f M_{t}+I=g M_{t-1}+I$ of the form

$$
0 \longrightarrow \begin{gathered}
R[-3 t+1] \\
\oplus \\
R[-3 t]
\end{gathered} \longrightarrow R[-2 t]^{2 t+2} \longrightarrow \begin{gathered}
R[-t]^{2} \\
\oplus \\
R[-2 t+1]^{2 t-1}
\end{gathered} \longrightarrow J \longrightarrow 0
$$

and

$$
0 \rightarrow \begin{gathered}
R[-3 t+1] \\
\oplus \\
\mathbb{F}_{3}[-t+1]
\end{gathered} \rightarrow \begin{gathered}
R[-2 t] \oplus R[-2 t+1]^{2} \\
\oplus \\
\mathbb{F}_{2}[-t+1]
\end{gathered} \rightarrow \begin{gathered}
\oplus[-t]^{2} \\
\oplus \\
\mathbb{F}_{1}[-t+1]
\end{gathered} \rightarrow J \rightarrow 0 .
$$

The first free resolution must be minimal, hence

$$
\mathbb{F}_{3} \supseteq R[-2 t-1], \mathbb{F}_{2} \supseteq R[-2 t]^{2 t+1} \text {, and } \mathbb{F}_{1} \supseteq R[-t]^{2 t+1} \text {. }
$$

Since no cancellation is possible among $\mathbb{F}_{1}[-t+1], \mathbb{F}_{2}[-t+1]$ and $\mathbb{F}_{3}[-t+1]$ in the second free resolution of $J$, we deduce that all the containments in (4.1) must be equalities.

\section{References}

[A] L. Avramov. "A class of factorial domains", Serdica 5 (1979), 378379 .

[BE] D. Buchsbaum, D. Eisenbud. "Algebra structures for finite free resolutions, and some structure theorems for ideals of codimension 3", Amer. J. Math. 99 (1977), no. 3, 447-485.

[BCG] N. Budur, M. Casanellas, E. Gorla. "Hilbert functions of irreducible arithmetically Gorenstein schemes", J. Algebra 272 (2004), no. 1, $292-310$.

[C] A. Conca, "Gröbner bases of ideals of minors of a symmetric matrix", J. Algebra 166 (1994), no. 2, 406-421.

[CN] A. Corso, U. Nagel. "Monomial and toric ideals associated to Ferrers graphs", Trans. Amer. Math. Soc. 361 (2009), no. 3, 1371-1395.

[D] E. De Negri. "Pfaffian ideals of ladders", J. Pure Appl. Alg.125 (1998), 141-153.

[D1] E. De Negri. "Some results on Hilbert series and $a$-invariant of Pfaffian ideals", Math. J. Toyama Univ. 24 (2001), 93-106.

[DGo] E. De Negri, E. Gorla. "G-Biliaison of ladder Pfaffian varieties", J. Algebra 321 (2009), no. 9, 2637-2649.

[K] C. Krattenthaler, "The major counting of nonintersecting lattice paths and generating functions for tableaux", Mem. Amer. Math. Soc. 115 (1995).

[HT] J. Herzog, N. V. Trung. "Gröbner bases and multiplicity of determinantal and Pfaffian ideals", Adv. Math. 96 (1992), 1-37.

[HTV] J. Herzog, N. V. Trung, G. Valla. "On hyperplane sections of reduced irreducible varieties of low codimension", J. Math. Kyoto Univ. 34 (1994), no. 1, 47-72. 
[KL] H. Kleppe, D. Laksov. "The algebraic structure and deformation of Pfaffian schemes", J. Algebra 64 (1980), 167-189. 